Background: The problem of sarcopenia (SP) in rheumatoid arthritis (RA) is particularly significant in terms of assessing the risk of fractures, since SP leads to falls, which are an independent risk factor for fractures along with RA and osteoporosis. Objectives: To evaluate the bone mineral density (BMD) and fracture risk in women with RA and SP.

Methods: 79 women with RA based on the 2010 ACR/EULAR classification criteria were included: 20 (25\%) women with confirmed SP (age median 59 [53; 64]) according to EWGSOP2 criteria and 59 (75\%) women without SP (age median 60 [55; 67]) ( $p>0.05)$. We assessed clinical data: age, body mass index (BMI), disease duration, anthropometric measurements, C-reactive protein level, disease activity score in 28 joints-erythrocyte sedimentation rate (DAS28-ESR), previous medication use including glucocorticoids and methotrexate, muscle strength and function. Dual-energy X-ray absorptiometry (DXA) to measure BMD of lumbar spine (LS), femoral neck (FN) and total hip (TH) was performed. The 10-year probability of major osteoporotic fracture (clinical spine, forearm, hip or shoulder fracture) and the 10-year probability hip fracture was calculated using the Russian version of the FRAX® tool. Statistical analysis was performed using non-parametric methods. All patients signed an informed consent to participate. Results: Median BMD in LS was $0.892[0.772 ; 1.024] \mathrm{g} / \mathrm{cm}^{2}$ in patients with SP and $0.910[0.785 ; 1.028] \mathrm{g} / \mathrm{cm}^{2}$ - without SP $(p>0.05)$. There was significant difference between groups in the proximal femur BMD: $0.760[0.731 ; 0.826] \mathrm{g} /$ $\mathrm{cm}^{2}$ in $\mathrm{TH}$ and $0.681[0.607 ; 0.703] \mathrm{g} / \mathrm{cm}^{2}$ in $\mathrm{FN}$ in patients with SP and 0.838 $[0.735 ; 0.921] \mathrm{g} / \mathrm{cm}^{2}$ in $\mathrm{TH}$ and $0.719[0.622 ; 0.804] \mathrm{g} / \mathrm{cm}^{2}$ in $\mathrm{FN}$ in patients without SP ( $p=0.009$ and $p=0.048$, respectively). The frequency of osteoporosis was $35 \%$ and $22 \%$ in patients with and without SP $(p>0,05)$. The 10-year probability of major osteoporotic fracture with / without femoral neck BMD data was $22,0 \%[17,0 ; 32,0] / 19,5 \%[18,5 ; 22,5$ and $13,3 \%[9,8 ; 18,5] / 12,8 \%[9,3 ; 17,0]$ in patients with SP and without SP $(\mathrm{p}<0.05)$ and the 10 -year probability of hip fracture with / without femoral neck BMD data - 3,1\% [3,0; 7,5] / 3,1\% $[2,3 ; 3,3]$ and $1,4 \%[0,9 ; 2,78] / 0,65[0,4 ; 1,7]$, respectively $(p<0.05)$.

Conclusion: There were no differences in the frequency of osteoporosis between patients with SP and without SP, however women with SP had proximal femur BMD less than women without SP. The probability of major osteoporotic fracture and hip fracture was significantly higher in patients with RA and SP compared with patients without SP.

Disclosure of Interests: None declared

DOI: 10.1136/annrheumdis-2020-eular.3066

\section{SAT0095 REAL LIFE SEVERE INFECTIONS IN PATIENTS WITH RHEUMATOID ARTHRITIS ON TREATMENT WITH BIOLOGICAL THERAPY AND JAKI}

L. Vega ${ }^{1}$, I. Calvo ${ }^{1}$, O. Ibarguengoitia ${ }^{1}$, J. M. Blanco ${ }^{1}$, D. Montero ${ }^{1}$, C. García ${ }^{1}$, M. E. Ruiz ${ }^{1}$, A. R. Inchaurbe ${ }^{1}$, I. Torre ${ }^{1}$, C. E. Perez ${ }^{1}$, O. Fernandez ${ }^{1}$, E. Cuande ${ }^{1}$, I. Gorostiza ${ }^{2}$, M. L. García Vivar ${ }^{1}$, E. Galindez ${ }^{1}{ }^{1}$ Basurto University Hospital, Rheumatology, Bilbao, Spain; ${ }^{2}$ Basurto University Hospital, Investigation, Bilbao, Spain

Background: Infections are one of the main complications among patients with rheumatoid arthritis (RA) with immunosuppressive treatment. The differences between treatments and the influence of other factors is unclear.

Objectives: To evaluate the frequency and factors associated with serious infections in patients with RA treated with biological therapy (BT) and JAKi and the differences between treatments.

Methods: Descriptive and retrospective study (January 2015-December 2019) of patients with RA treated with BT (TNFi, non-TNFi) and JAKi (tofacitinib, bariticinib) in a single center. Severe infection was considered a life-threatening infection or one that required hospitalization and intravenous treatment. Epidemiological variables, clinical characteristics, Charlson comorbidity index, type of BT or JAKi and concomitant treatment were collected.

For the analysis frequencies and percentages are used in qualitative variables, and mean $\pm S D$ in the quantitative ones. Statistical analysis was performed with IBM SPSS v 23.

Results: We registered 246 patients ( $85 \%$ women) mean aged $55.8 \pm 13.5$ years. RF was positive in $87 \%$, anti-CCP in $75.6 \%$ and $15.4 \%$ presented extra-articular manifestations (nodulosis $8.9 \%$, intersticial lung disease $5.3 \%$, other $1.2 \%$ ). At the start of the study 149 patients $(60.6 \%)$ were with TNFi, $79(32.1 \%)$ nonTNFi and $18(7.3 \%)$ with JAKi and non-biologic DMARD (nbDMARDs) were used in $84.1 \%$ of cases (methotrexate $71.2 \%$, leflunomide $21.4 \%$, other $7.4 \%$ ). During the study 176 patients $(71.5 \%)$ continued with the same treatment and in $70(28.5 \%)$ it was changed at least once. 5 patients discontinued the treatment. At the end of the study, 124 patients $(50.4 \%)$ were with TNFi, $83(33.7 \%)$ non-TNFi and $34(13.8 \%)$ with JAKi.

Severe infection was developed in $17(6.9 \%)$ patients (respiratory 7 , se sis 4 , urinary 3 , cellulitis 2 , osteomyelitis 1 ) among them 2 patients had severe infection and herpes zoster and 3 developed a second infection. 9 patients were with TNFi (52.9\%), 6 non- TNFi BT (35.3\%) and 2 JAKi (11.8\%). Table 1
TABLE 1. CHARACTERISTICS OF PATIENTS WITH INFECTION VS. WITHOUT INFECTION

\begin{tabular}{lccc}
\hline & INFECTION & & \\
\hline & YES n:17 & NO n:229 & p \\
\hline FEMALE, n (\%) & $13(76.5)$ & $196(85.6)$ & 0.297 \\
AGE years, (mean \pm SD) & $60.8 \pm 13$ & $55.4 \pm 13.5$ & 0.112 \\
AGE $\geq$ 65 n (\%) & $9(52.9)$ & $63(27.5)$ & 0.070 \\
RF +, n (\%) & $17(100)$ & $197(86)$ & 0.139 \\
ANTI-CCP +, n (\%) & $14(82.4)$ & $172(75.1)$ & 0.770 \\
ILD, n (\%) & $1(25)$ & $12(35.3)$ & 0.708 \\
ALCOHOL, n (\%) & $1(5.9)$ & $19(8.3)$ & 1.00 \\
SMOKER, n (\%) & $5(29.4)$ & $60(26.2)$ & 0.772 \\
COPD, n (\%) & $5(29.4)$ & $24(10.5)$ & $\mathbf{0 . 0 3 6 ^ { * }}$ \\
DM, n (\%) & $7(41.2)$ & $19(8.3)$ & $\mathbf{0 . 0 0 1 ^ { * }}$ \\
SEVERE LIVER DISEASE, n (\%) & $2(11.8)$ & $1(0.4)$ & $\mathbf{0 . 0 1 3 ^ { * }}$ \\
RENAL INSUFFICIENCY, n (\%) & $2(11.8)$ & $3(1.3)$ & $\mathbf{0 . 0 4 0}$ \\
PERIPHERAL VASCULAR DISEASE, n (\%) & $7(41.2)$ & $25(10.9)$ & $\mathbf{0 . 0 0 3 ^ { * }}$ \\
CHARLSON INDEX (mean $\pm S D)$ & $2.35 \pm 2.1$ & $0.66 \pm 1.2$ & $\mathbf{0 . 0 1 4 ^ { * }}$ \\
TNFi, n (\%) & $9(52.9)$ & $115(50.2)$ & \\
NON-TNFi $n(\%)$ & $6(35.3)$ & $77(33.6)$ & \\
JAKi, n (\%) & $2(11.8)$ & $32(14)$ & \\
nbDMARDs, n (\%) & $12(70.6)$ & $156(68.1)$ & 0.833 \\
GCC, n (\%) & $13(76.5)$ & $115(50.2)$ & $\mathbf{0 . 0 3 7 ^ { * }}$ \\
\hline
\end{tabular}

The inflammatory activity of RA was mild at the time of infection (DAS28: 2.7 \pm 1.2 ). The median time until infection was: TNFi 28.05 months, non- TNFi BT 25.03 and Jakinibs 16.97 .

The Charlson index, concomitant treatment with glucocorticoids (GCC) (not treatment with nbDMARDs), chronic obstructive pulmonary disease (COPD), diabetes (DM), severe liver disease and moderate-severe renal insufficiency were statistically significantly associated with infection. Table 1

Conclusion: In our study, $6.9 \%$ of patients with RA treated with BT or JAK developed severe infection during 4 years of follow-up. Concomitant GCC therapy and comorbidity increased the risk of presenting this complication.

Disclosure of Interests: None declared

DOI: 10.1136/annrheumdis-2020-eular.3691

\section{SAT0096 \\ DISCORDANCE BETWEEN SUBJECTIVE AND OBJECTIVE INDEX OF THE DISEASE ACTIVITY SCORE MAY REDUCE THE CORRELATION BETWEEN CLINICAL AND ULTRASOUND ASSESSMENT IN RHEUMATOID ARTHRITIS}

$\underline{\text { A. Xie }}^{1}$, L. Ji ${ }^{1}$, Z. Zhang ${ }^{1} .{ }^{1}$ Peking University First Hospital, Rheumatology and Clinical Immunology, Beijing, China

Background: There was discordance between subjective and objective index of the disease activity score, or between clinical parameters and ultrasound findings in some RA patients. Therefore, we set out to determine whether the discordance between subjective and objective index of the composite score could reduce the correlation between clinical and ultrasound parameters in RA

Objectives: To investigate whether the discordance between tender and swollen joint count (TJC and SJC) as well as patient's and evaluator's global assessment (PGA and EGA) influences the correlation between clinical and US parameters in RA.

Methods: RA patients with available ultrasonography of 28 joints from Jan 2014 to Jan 2018 were enrolled in the study. Gray-scale (GS) synovial hypertrophy and Power Doppler (PD) synovitis were measured and semi-quantitatively graded. The total GS/PD score was the sum score of 28 joints. SJC and TJC based on 28 joints, PGA and EGA of all the patients were evaluated by one rheumatologist. The numeric difference between TJC and SJC ( $\triangle T S J)$ and that between PGA and EGA ( $\triangle \mathrm{PEG}$ ) were calculated. The correlation between clinical and ultrasound parameters in different $\triangle \mathrm{TSJ}$ and $\triangle \mathrm{PEG}$ subgroups was explored.

Results: Totally 163 patients were enrolled in the study. Clinical composite disease activity scores and all the components were significantly correlated with the total GS and PD scores ( $<<0.01$ for all). But the relevance between the clinical disease parameters and total PD score became weak, with the increase of $\Delta$ TSJ. For the patients with $\Delta T S J>5$, the total PD score was only correlated with CRP, EGA and PGA, while the total GS score was only correlated with CRP. Similarly, no correlation between total PD score and clinical parameters, except for SJC, was observed in patients with $\triangle P E G<0$ ( $p<$ 0.05).

Conclusion: Total PD/GS score was correlated well with the clinical param eters of disease activity, including both the subjective and objective indexes. But for patients with $\triangle T S J>5$, there was no correlation between total GS/PD scores and clinical composite disease activity scores, except that only the objective index (CRP, SJC and EGA) were more likely to correlate with total GS/PD scores. 
Disclosure of Interests: None declared

DOI: 10.1136/annrheumdis-2020-eular.5740

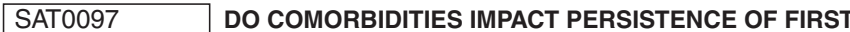 TUMOR NECROSIS FACTOR INHIBITOR TREATMENT IN RHEUMATOID ARTHRITIS? DATA FROM TURKBIO}

T. Yüce İnel ${ }^{1}$, S. B. Kocaer ${ }^{1}$, Y. Erez ${ }^{1}$, S. Gulle ${ }^{1}$, A. Karakas ${ }^{1}$, A. Köken Avşar ${ }^{1}$,

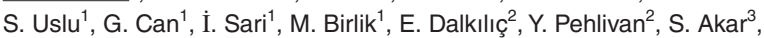
B. Goker ${ }^{4}$, G. Yıldırım Cetin ${ }^{5}$, S. Haznedaroglu ${ }^{4}$, Ş. Yavuz ${ }^{6}$, T. Pirildar ${ }^{7}$, H. Direskeneli ${ }^{8}$, N. Akkoc ${ }^{9}$, F. Onen ${ }^{1}$ on behalf of Turkbio Registry Group. ${ }^{1}$ Dokuz Eylul University Faculty of Medicine, İzmir, Turkey; ${ }^{2}$ Uludag University Faculty of Medicine, Bursa, Turkey; ${ }^{3}$ Katip Celebi University, İzmir, Turkey; ${ }^{4}$ Gazi University Faculty of Medicine, Ankara, Turkey; ${ }^{5}$ Sutcu Imam University, Kahramanmaras, Turkey; ${ }^{6}$ Bilim University, Istanbul, Turkey; ${ }^{7}$ Celal Bayar University, Manisa, Turkey; ${ }^{8}$ Marmara University, Istanbul, Turkey; ${ }^{9}$ Celal Bayar University, Manisa, Turkey

Background: Studies indicate that patients with rheumatoid arthritis (RA) are at increased risk of developing several comorbid disorders. Comorbidities affect treatment decisions, the effectiveness of the treatment, quality of life, RA prognosis, and survival rate [1]

Objectives: The aim of this study to investigate the impact of comorbidity on the first TNF inhibitor treatment persistence in RA.

Methods: In the TURKBIO database, patients with an ICD 10-diagnosis of RA (M05 or M06) who started TNF inhibitor therapy between January 2011 and June 2019 were enrolled. Demographic and clinical characteristics, acute phase reactants, disease activity scores (DAS $28 \mathrm{CRP}, \mathrm{HAQ}, \mathrm{CDAI}$, VAS global), initial comorbidities and numbers, drug persistence, were evaluated. Kaplan-Meier plots and Cox proportional hazard regression analyses were performed.

Results: A total of 1172 patients $>18$ years of age treated with TNF-a inhibitors were included in the study. The most prevalent comorbidities were: hypertension in 262 patients (32.6\%), obesity in 254 (32.6\%), osteoporosis in 178 $(22.3 \%)$, chronic lung disease in $143(17.9 \%)$ and depression in $126(15.8 \%)$. The baseline characteristics are summarised in Table 1. The presence of comorbidity did not affect the survival rate of the first TNF inhibitor therapy in the RA patients ( $p: 0.65)$. Comorbidities had no effect on DAS28 CRP (> 1.2 reduction) responses at 6 and 12 months of treatment ( $p: 0.18, p: 0.83$, respectively). As the mean disease duration increases, the persistence of the first TNF inhibitor decreases by $5 \%$.

Conclusion: This study demonstrated the increasing burden of comorbidities in RA. However, it suggested that the presence and number of comorbidities did not influence the rate of persistence in the first TNF inhibitor drug and the response to treatment.

Table 1 Characteristics of RA patients

\begin{tabular}{|c|c|}
\hline Female, n (\%) & 935 (79.8) \\
\hline Age, years ${ }^{\star}$ & $51.0 \pm 13.7$ \\
\hline Current smokers, n (\%) & $256(23.2)$ \\
\hline RF Positivity, n (\%) & $404(55.6)$ \\
\hline Anti-CCP Positivity, n (\%) & $430(58.2)$ \\
\hline X-ray Erosion, n (\%) & $317(61.9)$ \\
\hline $\mathrm{ESR}, \mathrm{mm} / \mathrm{h}^{\star}$ & $31.2 \pm 21.9$ \\
\hline CRP, mg/L* & $17.2 \pm 3.9$ \\
\hline DAS 28-CRP* & $3.8 \pm 1.6$ \\
\hline VAS global* & $46.6 \pm 28.6$ \\
\hline$H A Q^{*}$ & $0.9 \pm 0.7$ \\
\hline \multicolumn{2}{|l|}{ First TNFi } \\
\hline Etanercept, $\mathrm{n}(\%)$ & $525(38.7)$ \\
\hline Adalimumab, $\mathrm{n}(\%)$ & 379 (27.9) \\
\hline Infliximab, $\mathrm{n}(\%)$ & $118(8.7)$ \\
\hline Certolizumab, n (\%) & $188(13.8)$ \\
\hline Golimumab, $\mathrm{n}(\%)$ & $147(10.9)$ \\
\hline
\end{tabular}

* mean \pm S.D

RF, Rheumatoid factor; Anti-CCP, Anti- cyclic citrullinated peptid; ESR, erythrocyte sedimentation rate; CRP, C-reactive protein; DAS28-CRP, Disease Activity Score using 28 joints-CRP; VAS, Visual analog scale; HAQ, Health Assessment Questionnaire

\section{References:}

[1] Gabriel, S.E. and K. Michaud, Epidemiological studies in incidence, prevalence, mortality, and comorbidity of the rheumatic diseases. Arthritis research \& therapy, 2009. 11(3): p. 229.

Disclosure of Interests: None declared

DOI: 10.1136/annrheumdis-2020-eular.1599
SATURDAY, 06 JUNE 2020

Rheumatoid arthritis - biological DMARDs

\section{SAT0098 TREATMENT OF A COHORT OF PATIENTS WITH INTERSTITIAL LUNG DISEASE AND RHEUMATOID ARTHRITIS}

C. Aguilera $\mathrm{Cros}^{1}$, M. Gomez Vargas ${ }^{1}$, R. J. Gil Velez ${ }^{1}$, J. A. Rodriguez Portal ${ }^{2}$ ${ }^{1}$ University Hospital Virgen del Rocío, Department of Rheumatology, Seville, Spain; ${ }^{2}$ University Hospital Virgen del Rocío, Department of Pneumology, Seville, Spain

Background: There is no specific treatment for interstitial lung disease (ILD) secondary to Rheumatoid Arthritis (RA) other than the treatment of RA without extra-articular involvement. Current regimens usually include corticosteroid therapy with or without immunosuppressants (IS), there is no consensus for the treatment.

Objectives: To analyze the different treatment regimens in a cohort of patients with ILD and RA in our clinical practice.

Methods: Descriptive study of 57 patients treated in our Hospital (1/1/2018 unti 12/31/2019) with a diagnosis of RA (ACR 2010 criteria) and secondary ILD. The most recent American Thoracic Society (ATS)/European Respiratory Society (ERS)/Japanese Respiratory Society (JRS)/Latin American Thoracic Society (ALAT) guidelines define three HRCT (High Resolution Computed Tomography) patterns of fibrosing lung disease in the setting of idiopathic pulmonary fibrosis (IPF): definite Usual Interstitial pneumonia (UIP) (traction bronchiectasis and honeycombing), possible UIP and inconsistent with UIP. The distinction between definite UIP and possible UIP in these to the presence or absence of honeycombing. Approved by the Ethics Committee. Quantitative variables are expressed as mean (SD) and dichotomous variables as percentages (\%). Statistical analysis with SPSS version 21

Results: 21 men and 36 women were included, with a mean age of $69 \pm 10$ years (mean $\pm S D$ ), history of smoking (smokers $14 \%$, non-smokers $43 \%$, forme smokers $42 \%$ ). Clinical ILD at diagnosis (dyspnea 61\%, dry cough $56 \%$, crackling $70 \%$, acropachy $7 \%$ ). $84 \%$ were positive rheumatoid factor and $70 \%$ positive anticitrullinated protein antibody.

Diagnosis of ILD by HRCT in $100 \%$ of patients with different patterns: defined UIP 26 (45\%), probable UIP 2 (3\%) and not UIP 29 (50\%). The diagnosis of ILD was confirmed by biopsy in 12 patients.

$79 \%$ underwent $(\mathrm{T})$ treatment prior to the diagnosis of ILD with glucocorticoids and disease-modifying drugs (DMARD). Among the traditional DMARDs used were: Methotrexate $68 \%$ (there were no cases of MTX pneumonitis), Leflunomide $47 \%$, Hydroxychloroquine $26 \%$ and Sulfasalazine $21 \%$. Biological therapy in 15 patients: Etanercept 19\%, Adalimumab 5\%, Infliximab 3\% and Certolizumab $2 \%$. Two patients presented an exacerbation and rapid progression of the ILD during the T with Etanercept with the final result of death.

T with IS after the diagnosis of ILD in $80 \%$ of patients (Azathioprine 15, Rituximab 14, Abatacept 10, Tocilizumab 4, Sarilumab 1, Mofetil mycophenolate 1 and Cyclophosphamide 1).

Two patients with defined UIP perform T with antifibrotic: 1st Nintedanib (INBUILD Trial, This article was published on September 29, 2019, at NEJM.org) 2nd Pirfenidone (initial diagnosis of IPF Idiopathic Pulmonary Fibrosis and subsequent of seropositive RA with UIP). Both improved greater than $10 \%$ in forced vita capacity (FVC) and diffusion capacity of the lung for carbon monoxide (DLCO) in the 6 months after onset of $\mathrm{T}$.

Conclusion: Our results, in general, agree with what is published in the literature. Prospective, multicentre and larger sample studies are necessary to bette define which patients would benefit more from IS T or antifibrotic T (or if the antifibrotic should be added to the previous IS).

Disclosure of Interests: None declared DOI: 10.1136/annrheumdis-2020-eular.4907

\section{\begin{tabular}{|l|l}
\hline SAT0099 BMI AND TREATMENT SURVIVAL IN RA PATIENTS \\
\hline
\end{tabular} STARTING TREATMENT WITH TNFA-INHIBITORS: LONG TERM FOLLOW-UP IN THE REAL LIFE METEOR REGISTRY}

S. A. Bergstra ${ }^{1}$, D. Vega-Morales ${ }^{2}$, E. Murphy ${ }^{3}$, M. De Buck ${ }^{4}$, K. SolomonEscoto $^{5}$, T. Huizinga ${ }^{1}$, C. Allaart ${ }^{1} .{ }^{1}$ LUMC, Rheumatology, Leiden, Netherlands; ${ }^{2}$ Universidad Autónoma de Nuevo León, Rheumatology, Monterrey, Mexico; ${ }^{3}$ University Hospital Wishaw, Wishaw, United Kingdom; ${ }^{4}$ Haaglanden Medical Center, Rheumatology, The Hague, Netherlands; ${ }^{5}$ University of Massachusetts Medical School, UMass Memorial Medical Center, Rheumatology, Worcester, United States of America

Background: BMI appears to be associated with treatment response on TNFi(nhibitors) in rheumatoid arthritis (RA), but large heterogeneity between studies exists. More extreme BMI categories are rarely studied and it is unclear if differences exist between various TNFi. ${ }^{1}$ 